\title{
Neurology
}

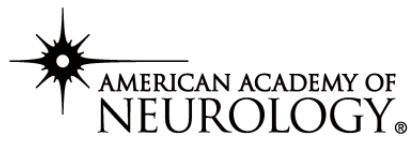

The most widely read and highly cited peer-reviewed neurology journal

The Official Journal of the American Academy of Neurology

Neurology Publish Ahead of Print

DOI: 10.1212/WNL.0000000000011746

\section{Cerebral Small Vessel Disease and Functional Outcome Prediction after}

\section{Intracerebral Haemorrhage}

${ }^{*} \mathrm{IC}$ Hostettler $\mathrm{MD}^{1},{ }^{*} \mathrm{G}$ Schwarz $\mathrm{MD}^{1,2}$, G Ambler $\mathrm{PhD}^{3}$, D Wilson $\mathrm{PhD}^{1}$, G Banerjee PhD, MRCP, $\mathrm{D}$ Seiffge MD ${ }^{1,4}$, C Shakeshaft $\mathrm{MSc}^{1}$, S Lunawat ${ }^{1}, \mathrm{H}$ Cohen MD, FRCP ${ }^{5}$, T Yousry, FRCR ${ }^{6}$, R Al-Shahi Salman $\mathrm{PhD}^{7}$, GYH Lip FRCP ${ }^{8}$, MM Brown FRCP ${ }^{1}$, KW Muir MD, FRCP ${ }^{9}, \mathrm{H}_{\text {Houlden }} \mathrm{MRCP}^{10}$, HR Jäger MD, FRCR ${ }^{6}$, DJ Werring PhD, FRCP ${ }^{1}$; on behalf of the CROMIS-2 collaborators.

Neurology® Published Ahead of Print articles have been peer reviewed and accepted for publication. This manuscript will be published in its final form after copyediting, page composition, and review of proofs. Errors that could affect the content may be corrected during these processes. 
*These authors contributed equally

${ }^{1}$ Stroke Research Centre, University College London, Queen Square Institute of Neurology, London, UK 2Department of Neurology, Stroke Unit San Raffaele Hospital, Milan, Italy

${ }^{3}$ Department of Statistical Science, University College London, Gower Street, London, UK

${ }^{4}$ Department of Neurology and Stroke Center, Inselspital, Bern, Switzerland

${ }^{5}$ Haemostasis Research Unit, Department of Haematology, University College London, 51 Chenies Mews, London, UK

${ }^{6}$ Lysholm Department of Neuroradiology and the Neuroradiological Academic Unit, Department of Brain Repair and Rehabilitation, UCL Institute of Neurology, Queen Square, London, UK

${ }^{7}$ Centre for Clinical Brain Sciences, School of Clinical Sciences, University of Edinburgh, Edinburgh, UK

${ }^{8}$ Liverpool Centre for Cardiovascular Science, Liverpool Heart and Chest Hospital, University of Liverpool, Liverpool, United Kingdom

${ }^{9}$ Institute of Neuroscience \& Psychology, University of Glasgow, Queen Elizabeth University Hospital, Glasgow, UK

${ }^{10}$ Department of Molecular Neuroscience, UCL Institute of Neurology and the National Hospital for Neurology and Neurosurgery, Queen Square, London

Article word count: 3154

Abstract word count: 250

Title character count: 95

Figure count: 2

Table count: 5

Reference count: 30

Supplemental Data: doi:10.5061/dryad.ksn02v72s

Corresponding author:

Professor David Werring

Email: d.werring@ucl.ac.uk. 
Statistical analysis conducted by Ghil Schwarz (MD), Isabel C Hostettler (MD) and Gareth Ambler (PhD), University College London.

Sources of funding: DJW and DW receive funding from the Stroke Foundation/British Heart Foundation. This work was undertaken at UCLH/UCL which receives a proportion of funding from the Department of Health's National Institute for Health Research (NIHR) Biomedical Research Centres funding scheme. GB holds an NIHR Academic Clinical Fellowship and has received funding from the Rosetrees Trust.

Disclosures: H.C. reports grants and other support from Bayer Healthcare and UCB outside the submitted work. T.Y. reports personal fees and other support from GlaxoSmithKline, Biogen Idec, Novartis, ESOR, Merck, Hikma and Parexel outside the submitted work. G.Y.H.L. reports consultancy and speaker fees from Bayer, Bayer/Janssen, BMS/Pfizer, Biotronik, Medtronic, Boehringer Ingelheim, Microlife, Roche and Daiichi $\square$ Sankyo outside the submitted work; no fees are directly received personally. K.W.M. reports personal fees from Bayer, personal fees and non $\square$ financial support from Boehringer Ingelheim and personal fees from Daiichi@Sankyo outside the submitted work. DJS: Scientific advisory boards: Bayer and Pfizer. Compensation for educational efforts: Stago. DJS is supported by a fellowship from the Swiss National Science Foundation (SNF), the Bangerter-Rhyner Foundation, the Bayer Foundation 2017 "Thrombosis Research Award" and the Swiss Society of Neurology. D.J.W. reports personal fees from Bayer, Alnylam and Portola outside the submitted work. The remaining authors declare no financial or other conflicts of interest.

Keywords. Intracerebral haemorrhage, long-term functional outcome, small vessel disease, CT

Coinvestigator Appendix [AR 1.22.2021] --- http://links.lww.com/WNL/B345 


\section{ABSTRACT}

Objective. To determine whether CT-based cerebral small vessel disease (SVD) biomarkers are associated with 6-month functional outcome after intracerebral hemorrhage (ICH), and whether these biomarkers improve the performance of pre-existing ICH score.

Methods. We included 864 patients with acute ICH from a multicentre, hospital-based prospective cohort study. We evaluated CT-based SVD biomarkers (white matter hypodensities [WMH]; lacunes; brain atrophy; and a composite SVD burden score) and their associations with poor 6-month functional outcome (modified Rankin Scale $[\mathrm{mRS}]$ score $>2$ ). The area under the receiver operating characteristic curve (AUROC) and Hosmer-Lemeshow test were used to assess discrimination and calibration of the ICH score with and without SVD biomarkers. Results. In multivariable models (adjusted for ICH score components), WMH presence (OR 1.52, 95\%CI 1.12-2.06), cortical atrophy presence (OR 1.80, 95\%CI 1.19-2.73), deep atrophy presence (OR 1.66, 95\%CI 1.17-2.34), and severe atrophy (either deep or cortical) (OR 1.94, 95\%CI 1.36-2.74) were independently associated with poor functional outcome. For the ICH score, the AUROC was 0.71 (95\%CI 0.68-0.74). Adding SVD markers did not significantly improve ICH score discrimination; for the best model (adding severe atrophy) the AUROC was 0.73 (95\%CI 0.69-0.76). These results were confirmed when considering lobar and non-lobar ICH, separately.

Conclusions. The ICH score has acceptable discrimination for predicting 6-month functional outcome after ICH. CT biomarkers of SVD are associated with functional outcome but adding them does not significantly improve ICH score discrimination. 


\section{INTRODUCTION}

Spontaneous (non-traumatic) intracerebral haemorrhage (ICH) is a devastating form of stroke, with $40 \%$ mortality at 30 days and $65 \%$ at one year [1], [2]. Only about $20 \%$ of survivors are functionally independent at 6 months [3], [4]. In order to plan clinical care and provide prognostic information, it is essential to understand which baseline factors, available in the acute phase, are associated with functional outcome. Several clinical-radiological scores have been developed to predict functional outcome or mortality after ICH at different time-points (usually 30 or 90 days). Subsequent studies have validated existing scores for different outcomes at different time-points[5]. The ICH score [6] (Table e-1) is the most commonly used and extensively validated predictive score [5]. The score (range 0-6) is the sum of points assigned to five variables: Glasgow Coma Scale score $3-4$ (2 points) or 5-12 (1 point); age $\geq 80$ years (1 point); infratentorial site (1 point); ICH volume $\geq 30 \mathrm{~mL}$ (1 point); and intraventricular hemorrhage (1 point).

Cerebral small-vessel disease (SVD) includes deep perforator (hypertensive) arteriopathy and cerebral amyloid angiopathy (CAA), which are considered extremes along a continuum of agerelated pathologies[7]. SVD causes around 77\% of spontaneous $\mathrm{ICH}[8]$, can readily be assessed using neuroimaging markers on both magnetic resonance imaging (MRI) and computed tomography (CT) scans, and is associated with functional outcome and cognitive decline[9] after stroke[10]. It is not known whether SVD burden can improve the prediction performance of existing clinical-radiological scores for functional outcome after ICH.

In a large multicentre UK ICH cohort, we aimed to investigate the association of CT-based SVD biomarkers with 6-month functional outcome and to assess whether these improve the predictive performance of the ICH score. 


\section{MATERIAL AND METHODS}

\section{Study population}

We included data from the CROMIS-2 (ICH), a prospective cohort study of adult patients with spontaneous, non-traumatic ICH (NCT02513316) undertaken at 79 UK hospitals (and one in the Netherlands). We included participants with: (1) all variables required for calculation of the ICH score (Glasgow Coma Score [GCS], ICH volume, ICH location [supratentorial or infratentorial], age [ $\geq 80$ or $<80$ ], and presence of intraventricular haemorrhage); (2) functional outcome recorded at 6 months using the modified Rankin Scale (mRS, dichotomized into 0-2 [good functional outcome] and 3-6 [poor functional outcome]); and (3) baseline acute brain CT of adequate quality to evaluate all SVD biomarkers. We excluded patients with a known underlying structural cause for ICH (arteriovenous malformation, tumour, cavernomas, intracranial aneurysm, or haemorrhagic transformation of an infarct). We collected detailed data on demographics, risk factors, clinical presentation and brain imaging findings. Standard Protocol Approvals, Registrations and patient consents. Written informed consent was obtained from all participants; in case of lack of capacity written informed consent was obtained from a relative or representative.

Ethical approval. The CROMIS-2 study was approved by the local Ethics Committee (reference: 10/H0716/64).

CT variables: selection, measurement and categorization Measurement of ICH volume was performed via a semi-automated (threshold-based) approach, as previously described[11]. ICH location was assessed using the Cerebral Haemorrhage Anatomical Rating Instrument (CHARTS)[12]. Two experienced trained raters (DS and DW) 
assessed the presence and severity of CT-based markers of SVD[13], [14], [15] [16] including: white matter hypodensities (WMH), lacunes, cortical atrophy, deep atrophy and SVD burden. WMH (also termed leukoaraiosis) were rated according to the Van Swieten Scale[17] which combines posterior (range $0-2$ ) and anterior (0-2) scores into an ordinal scale (0-4). WMH were considered severe if the Van Swieten scale was $\geq 2$ in either anterior or posterior periventricular white matter. WMH were dichotomized as: 1) present (Van Swieten $\geq 1$ [either anterior or posterior]) vs absent (Van Swieten = 0); and 2) severe vs non-severe. Lacunes were defined as round/ovoid, subcortical, fluid-filled cavities of 3-15 mm diameter, consistent with a previous infarct or haemorrhage[13]; $\geq 2$ lacunes were considered severe [14]. As previously described[14] [15] [16], we evaluated deep (enlargement of the ventricles) and superficial (enlargement of the sulci) cerebral atrophy using a template based three-point scale (absent/mild, moderate and severe). Atrophy was considered severe if it was graded as severe in either deep or cortical regions. A SVD burden score was calculated as previously described[14]: one point was assigned for: (1) severe WMH; (2) severe ( $\geq 2$ ) lacunes; and (3) presence of severe deep or cortical atrophy, giving a 4-point ordinal score (0-3). To evaluate the rater accuracy and reliability of CT scan assessment, both raters independently rated a random sample of $50 \mathrm{CT}$ scans. The SVD burden score was considered severe if the score was $\geq 2$. All ratings were blinded to outcome and all other patient clinical information.

\section{Statistical analysis}


We described categorical variables with frequencies and percentages, and continuous variables using mean and standard deviation (SD) or median with interquartile range (IQR). In univariable analysis, we used Chi-square and Wilcoxon/Mann-Whitney tests as appropriate. We first investigated the predictive performance of the ICH score and whether this could be improved through simple re-calibration (linear transformation of the predicted log-odds achieved through logistic regression). Next, we considered whether more complicated recalibration was necessary (re-estimation of all model coefficients using logistic regression). We evaluated associations between all radiological and clinical variables (components of the ICH score as well as all CT-based SVD markers) with 6-month functional outcome using univariable analysis. We then constructed multivariable models for the associations of each SVD biomarker individually, and for a SVD burden score, adjusted for ICH score variables (GCS, ICH volume, ICH location [supratentorial or infratentorial], age [ $\geq 80$ or $<80$ ], and presence of intraventricular haemorrhage) with poor 6-month functional outcome.

We then investigated whether we could improve the predictive performance for functional outcome of the ICH score through sequential addition of SVD markers found to be significantly associated with outcome in the univariable analysis; this analysis was performed in the entire cohort and in lobar and in non-lobar cohorts, separately. We also did a a sensitivity analysis using $\mathrm{mRS}>3$ as the definition of poor functional outcome.

Discrimination and calibration were assessed for each predictive model. We assessed discrimination by calculating the area under the receiver operating characteristic curve (AUROC). Calibration refers to the ability of a score to accurately predict the percentage of patients with the outcome of interest[18]. Inter-rater accuracy was evaluated via the proportion of agreement and Cohen's kappa statistic. We assessed calibration using the Hosmer-Lemeshow 
(HL) goodness-of-fit statistic: p-value $<0.05$ is suggestive of poor calibration. We adjusted all AUROC results for optimism, using the bootstrap. The significance level was set at $\mathrm{p}=0.05$. Statistical analysis was performed using STATA 16 (StataCorp. 2019. Stata Statistical Software: Release 16. College Station, TX: StataCorp LP).

Data Availability. All data requests should be submitted to the corresponding author for consideration by the CROMIS-2 Steering Committee. Supplementary data (Tables e-1-e-3) ) are available from Dryad https://doi.org/10.5061/dryad.ksn02v72s.

\section{RESULTS}

Of the original cohort of patients included in the CROMIS-2(ICH) study with available CT scan ( $\mathrm{n}=1037)$, we excluded 173 patients from our analysis: 52 did not have all variables needed to calculate the ICH score, and 121 did not have 6-month follow-up data (Figure 1). We included 864 patients; Table 1 summarizes the patient characteristics, outcomes and imaging variables. There were no statistically significant differences between included and excluded patients for any of the variables of interest (data not shown). Mean age was 73.6 years (SD 11.9). The majority of patients suffered from hypertension (580; 67.1\%). 429 patients had a deep ICH (49.6\%), 361 (41.8\%) lobar ICH and 74 (8.6\%) infratentorial ICH. The mean ICH volume was $14.9 \mathrm{~cm}^{3}\left(\mathrm{SD} 20.2 \mathrm{~cm}^{3}\right)$, and $256(29.6 \%)$ of patients had accompanying intraventricular haemorrhage. WMH was severe in 261 (30.2\%) of patients, SVD burden score was moderate-tosevere in 124 (14.3\%) patients, while 258 (29.9\%) had severe atrophy. 535 patients $(61.9 \%)$ had poor functional outcome $(\mathrm{mRS}>2)$ at 6 months. Inter-rater reliability for SVD biomarkers was as follows: atrophy $84.0 \%$ agreement, Cohen's kappa 0.68 ; WMH 79.6\% agreement, Cohen's kappa 0.52; lacunes 95.0\% agreement, Cohen’s kappa 0.45 . 


\section{Discrimination and calibration of the ICH score}

The discrimination of the ICH score for functional outcome at 6 months, measured by the AUROC, was 0.70 (95\% CI 0.67-0.74) (Figure 2A). After simple re-calibration, good agreement between observed and expected event rate was achieved (HL p = 0.26) (Figure 2B). After recalculation of $\beta$-coefficients for each variable included in the score (Table 2), the revised model demonstrated an AUROC of 0.71 (95\% CI 0.68-0.74). This new model showed improved calibration (HL p = 0.94).

Association between clinical-radiological variables and 6-month outcome

In the univariable analysis (Table 3), all variables included in the ICH score were associated with 6-month functional outcome, except for ICH location (supratentorial vs infratentorial, $\mathrm{p}=$ 0.296). With regards to CT biomarkers of SVD, WMH presence (OR 1.80, 95\% CI 1.36-2.37, p $<0.001$ ), severe WMH (OR 1.48, 95\% CI 1.09-2.01, p = 0.012), cortical atrophy (moderate OR 1.88, 95\% CI 1.29-2.76, $\mathrm{p}=0.001$; severe OR 4.41, 95\% CI 2.65-7.35, $\mathrm{p}<0.001$ ), deep atrophy (moderate OR 1.70, 95\% CI 1.23-2.35, $\mathrm{p}=0.001$; severe OR 3.32, 95\% CI 2.16-5.11, $\mathrm{p}<0.001$ ), severe atrophy (OR 2.36, 95\% CI 1.70-3.26, p <0.001) and severe SVD burden (OR 1.84, 95\% CI 1.20-2.81, $\mathrm{p}=0.004)$ were all associated with 6-month functional outcome.

After adjusting for variables in the ICH score (Table 4), presence of WMH (OR 1.52, 95\% CI 1.12-2.06, $\mathrm{p}=0.007)$, cortical atrophy (OR 1.80, 95\% CI 1.19-2.73, $\mathrm{p}=0.006)$, deep atrophy (OR 1.66, 95\%CI 1.17-2.34, $\mathrm{p}=0.004)$, severe atrophy (OR 1.94, 95\% CI 1.36-2.7, $\mathrm{p}<0.001)$ were associated with 6-month functional outcome. In adjusted analysis, severe SVD burden score showed a non-significant association with 6-month outcome (OR 1.57 [95\%CI 0.99-2.48]; p = 
0.053). The presence of severe atrophy was strongly associated with 6-month functional outcome, even after adjusting for other severe SVD markers and variables present in the ICH score (OR 2.22, 95\% CI 1.41-3.52, $\mathrm{p}=0.001)$.

\section{Addition of CT-based makers of SVD to ICH score}

When individually added to the revised ICH score model, WMH presence, cortical atrophy, deep atrophy, severe atrophy and severe SVD burden score did not significantly improve discrimination, as shown in Table 5; although an increase in the AUC was noted, but the 95\% confidence intervals for the AUROC all overlapped with those of the ICH score alone. The highest discrimination was achieved when adding severe atrophy with an AUROC of 0.73 (95\%CI 0.69-0.76).

\section{Sensitivity analysis}

In a sensitivity analysis using $\mathrm{mRS}>3$ as the definition of poor functional outcome, the discrimination of the ICH score for $\mathrm{mRS}>3$ was slightly better than for $\mathrm{mRS}>2$ but the $95 \%$ confidence intervals overlapped $(0.73$ [95\%CI 0.70-0.77] compared to 0.71 [95\%CI 0.68-0.74], respectively; Table e-2).

Separate analyses for lobar and non-lobar ICH

We conducted separate analyses on lobar and non-lobar ICH separately (Table e-3A, e-3B, e3C). This analysis confirmed the association between SVD biomarkers and outcome in both groups. In particular, in multivariable analysis cortical atrophy presence (OR 2.06 [95\%CI 1.213.52]; $\mathrm{p}=0.008)$, severe atrophy (OR 2.03 [95\%CI 1.30-3.17]; $\mathrm{p}=0.002)$ and severe SVD burden (OR 1.87 [95\%CI 1.03-3.39]; $\mathrm{p}=0.041)$ were strongly associated with outcome in non-lobar ICH (Table e-3D), while white matter hypodensities (OR 1.98 [95\%CI 1.21-3.24]; p = 0.006), deep atrophy presence (OR 1.97 [95\%CI 1.14-3.39]; $\mathrm{p}=0.014)$ and severe white matter 
hypodensities (OR 2.04 [95\% CI 1.18-3.53]; p = 0.011) were associated with outcome in lobar ICH (Table e-3E). The revised oICH score performed slightly better in lobar ICH (AUC 0.72 [95\%CI 0.67-0.77]), than in non-lobar ICH (AUC 0.69 [95\%CI 0.65-0.73]) but with overlapping 95\% CI. Adding SVD biomarkers to revised oICH score did not significantly improved outcome prediction of the oICH score alone (Table e-3F and e-3G). The best models for non-lobar ICH were obtained by adding cortical atrophy presence (AUC 0.71 [95\%CI 0.66-0.75]) or severe atrophy presence (AUC 0.71 [95\%CI 0.67-0.75]) to the score. For lobar ICH, the best model included the revised oICH score plus deep atrophy presence or oICH plus white matter hypodensities presence (in both models: AUC 0.74 [95\%CI 0.69-0.79]).

\section{DISCUSSION}

Our study has confirmed acceptable discrimination and calibration for the prediction of 6month functional outcome after ICH using a recalibrated version of the ICH score. We confirmed that some baseline CT biomarkers of SVD presence and severity are independently associated with 6-month functional outcome in univariable and multivariable models (adjusted for variables included in the ICH score). However, adding SVD biomarkers individually (or as a composite SVD burden score) did not lead to statistically significant improvement in the discriminative performance of the ICH score, regardless of ICH location.

Although many clinical-radiological scores have been developed, the ICH score remains widely used and validated, and performs well compared to others[5]. Four studies evaluated the performances of the ICH score for 6-months functional outcome prediction, and four studies for 12-months (pooled AUROC [0.78, 95\% CI 0.74-0.82]; pooled AUROC 0.77 [95\% CI 0.72-0.83], respectively). The discrimination of the ICH score in our study was not as high, although the 
95\% CI overlapped with that reported in previous studies. This might be due to differences in population characteristics and definitions of functional outcome. Previous studies included an Asian population [19], exclusively supratentorial ICH [20], or deep ICH with intraventricular extension[21]. Our population is likely to be more heterogeneous and therefore more generalizable to the full population of patients with $\mathrm{ICH}$. We used $\mathrm{mRS}>2$ to define poor functional outcome, but due to the severity of ICH, many studies use a cutoff of $\mathrm{mRS}>3$; our sensitivity found that the discrimination of the ICH score for $\mathrm{mRS}>3$ was slightly better than for $\mathrm{mRS}>2$ (0.73 [95\%CI 0.70-0.77] compared to 0.71 [95\%CI 0.68-0.74], respectively).

However, regardless of how poor outcome was defined, the addition of SVD biomarkers did not significantly improve predictive discrimination of ICH score alone.

Our findings that SVD markers are related to functional outcome suggest that SVD is an important factor in neurological recovery after ICH, in line with previous studies showing an association between baseline imaging features of SVD and cognitive impairment or functional outcome after ischemic and haemorrhagic stroke[22], [23]. The main reported associations are for 90-day outcome after ischemic stroke, and we are not aware of previous reports describing the impact of CT-based SVD markers on functional outcome beyond 3 months after ICH. Our results confirm previous data suggesting that $\mathrm{WMH}[10]$ and brain atrophy[24] are key factors through which SVD influences functional outcome. SVD could influence poor functional outcome via several mechanisms. First, SVD is likely to be associated with recurrent stroke after ICH; since most ICH are due to SVD, markers of its severity are likely to be associated with ICH recurrence risk. Second, SVD is associated with other vascular risk factors that could influence adverse vascular events. Third, SVD can lead to disruption of key brain networks likely to be 
important for rehabilitation, learning, and cognitive reserve [25], which are important in determining functional recovery after ICH.

However, we found that adding CT markers to the ICH score did not increase ICH score predictive discrimination significantly. The optimal discrimination was obtained when adding severe atrophy to the revised ICH score, but this small improvement was not statistically significant. Our findings thus indicate that although CT biomarkers of SVD (using visual rating scales) are clearly relevant for understanding mechanisms of functional recovery after ICH, adding them to the ICH score is not likely to be helpful in clinical practice for predicting of 6month outcome because it does not increase the predictive value of simple clinical-radiological characteristics of the index haemorrhagic event (age, ICH volume, location, intraventricular extension, conscious level). These findings were confirmed when considering lobar and nonlobar ICH separately; adding SVD biomarkers to the score did not significantly improve outcome prediction.

Our study measured functional outcome at a relatively early 6-month time point. Patients with acute ICH often have severe morbidity as a result of the haematoma, associated oedema, and intraventricular haemorrhage; these forms of brain injury seem to require a substantial time to resolve, meaning that functional improvement can occur beyond 6 months[26] [27] Thus, if SVD markers do influence ICH functional recovery, for example by impairing white matter connectivity (which might affect learning, general cognitive functioning, cognitive reserve and rehabilitation potential), they may not have additional influence over the initial ICH severity at the 6-month time-point, but could be a more important determinant of longer term outcome. CT brain scanning is a standard of care for patients with acute $\mathrm{ICH}$ that is readily available in most stroke services worldwide. Thus, our findings are relevant for clinical services and future 
research studies in large populations. Magnetic resonance imaging (MRI) has high sensitivity and specificity for detecting most manifestation of SVD[13], but is less accessible in many healthcare systems and is unsuitable for some patients (e.g. those who are unwell, claustrophobic, or have non-MRI compatible implants or devices). Nevertheless, further studies should assess the contribution of SVD markers assessed on MRI in improving outcome prognostication after ICH. Quantitative methods which can assess brain tissue microstructure and connectivity could be of particular interest; for example, diffusion tensor imaging techniques show promise for outcome prediction after both ischemic and hemorrhagic stroke[28], [29].

Our study has strengths. We evaluated a prospectively collected large cohort of ICH patients using a standardized protocol with good inter-rater agreement (with Cohen's kappa values comparable to previous studies). To the best of our knowledge this is the first attempt to improve outcome prognostication by adding CT-based markers of SVD to an existing clinicalradiological prognostic score. The visual rating methods we applied to quantify SVD burden on CT scans have been previously established[14] and can be applied to standard clinical CT scans that are usually part of standard clinical care. Given our multicenter design and large population cohort, with a range of ICH location and size, we expect that our results are generalizable, at least among the Western population. Our finding of lobar ICH in $41.8 \%$ of patients is consistent with previous population-based studies [30], suggesting that our cohort is generalizable to other ICH populations.

Our study also has limitations. The CROMIS-2 study required signed informed consent, which could have created a selection bias towards ICH survivors, limiting the inclusion of extremely severe ICH patients; this probably contributed to low rate of death at 6 months in our cohort. 
However, data from the group of ICH patients who are likely to survive the acute phase is of most relevance to clinicians, patients and their families. Our findings also require validation in independent cohorts from other populations.

\section{CONCLUSION}

The ICH score demonstrated acceptable prognostication ability for 6-months functional outcome after spontaneous ICH. We found that CT-markers of SVD were associated with 6month functional outcome when assessed independently in adjusted analyses, suggesting that they are relevant for ICH recovery mechanisms. However, adding them to the ICH score did not substantially improve predictive discrimination for 6-month functional outcome. Further studies investigating the influence of SVD markers on longer term outcome, and exploration of more advanced quantitative MRI methods are needed. 
Appendix 1: Authors

\begin{tabular}{|c|c|c|}
\hline Name & Location & Contribution \\
\hline Isabel C Hostettler & $\begin{array}{l}\text { University College } \\
\text { London }\end{array}$ & $\begin{array}{l}\text { Design of study; analysis and interpretation of } \\
\text { data; drafting the manuscript for intellectual } \\
\text { content. }\end{array}$ \\
\hline Ghil Schwarz & $\begin{array}{l}\text { University College } \\
\text { London }\end{array}$ & $\begin{array}{l}\text { Design of the study; analysis and interpretation } \\
\text { of data; drafting the manuscript for intellectual }\end{array}$ \\
\hline Gareth Ambler & $\begin{array}{l}\text { University College } \\
\text { London }\end{array}$ & $\begin{array}{c}\text { Analysis of data; revising manuscript for } \\
\text { intellectual content }\end{array}$ \\
\hline Duncan Wilson & $\begin{array}{l}\text { University College } \\
\text { London }\end{array}$ & $\begin{array}{l}\text { Analysis of data; revising manuscript for } \\
\text { intellectual content }\end{array}$ \\
\hline Gargi Banerjee & $\begin{array}{c}\text { University College } \\
\text { London }\end{array}$ & $\begin{array}{l}\text { Analysis of data; revising manuscript for } \\
\text { intellectual content }\end{array}$ \\
\hline David J Seiffge & University College & Revising manuscript for intellectual content \\
\hline Clare Shakeshaft & $\begin{array}{l}\text { University College } \\
\text { London }\end{array}$ & $\begin{array}{c}\text { Major role in acquisition of data; revising } \\
\text { manuscript for intellectual content }\end{array}$ \\
\hline Surabhika Lunawat & $\begin{array}{l}\text { University College } \\
\text { London }\end{array}$ & Revising manuscript for intellectual content \\
\hline Hannah Cohen & $\begin{array}{l}\text { University College } \\
\text { London }\end{array}$ & Revising manuscript for intellectual content \\
\hline Tarek A Yousry & $\begin{array}{c}\text { University College } \\
\text { London }\end{array}$ & Revising manuscript for intellectual content \\
\hline Rustam Al-Shahi & University of & Revising manuscript for intellectual content \\
\hline
\end{tabular}




\begin{tabular}{|r|c|c|}
\hline Salman & Edinburgh & \\
\hline Gregory Y H Lip & University of Liverpool & Revising manuscript for intellectual content \\
\hline Martin M Brown & University College & Revising manuscript for intellectual content \\
& London & \\
\hline Keith W Muir & University of Glasgow & Revising manuscript for intellectual content \\
\hline Hans Rolf Jäger & University College & Analysis of data; revising manuscript for \\
& London & intellectual content \\
\hline Henry Houlden & University College & Revising manuscript for intellectual content \\
& London & Study conceptualisation and design; \\
\hline David J Werring & University College & interpretation of data; revising manuscript for \\
& London & intellent content \\
\hline
\end{tabular}




\section{REFERENCES}

[1] ML Flaherty, M Haverbusch, P Sekar et al., "Long-term mortality after intracerebral hemorrhage," pp. 1-6, Mar. 2006.

[2] CJ van Asch, MJA Luitse, GJE Rinkel, I van der Tweel, A Algra, CJM Klijn, "Incidence, case fatality, and functional outcome of intracerebral haemorrhage over time, according to age, sex, and ethnic origin: a systematic review and meta-analysis," The Lancet Neurology, vol. 9, no. 2, pp. 167-176, Feb. 2010.

[3] L Tatu, T Moulin, R El Mohamad, F Vuillier, L Rumbach, A Czorny, "Primary Intracerebral Hemorrhages in the Besançon Stroke Registry,” pp. 1-6, May 2000.

[4] S Moulin, J Labreuche, S Bombois et al., "Dementia risk after spontaneous intracerebral haemorrhage: a prospective cohort study," The Lancet Neurology, vol. 15, no. 8, pp. 820-829, Jun. 2016.

[5] T Gregório, S Pipa, P Cavaleiro et al., "Assessment and Comparison of the Four Most Extensively Validated Prognostic Scales for Intracerebral Hemorrhage: Systematic Review with Metaanalysis," Neurocritical Care, vol. 30, no. 2, pp. 449-466, Nov. 2018.

[6] JC Hemphill, DC Bonovich, L Besmertis, GT Manley, C Johnston, “The ICH Score” Stroke, 2001 Apr;32(4):891-7

[7] S Schreiber, A Wilisch-Neumann, F Schreiber et al., "Invited Review: The spectrum of ageDrelated small vessel diseases: potential oyerlap and interactions of amyloid and nonamyloid vasculopathies," Neuropathol Appl Neurobiol, vol. 36, no. 3, pp. 158-21, Sep. 2019.

[8] NA Hilkens, CJJ van Asch, DJ Werring et al., "Predicting the presence of macrovascular causes in non-traumatic intracerebral haemorrhage: the DIAGRAM prediction score," J Neurol Neurosurg Psychiatry, vol. 89, no. 7, pp. 674-679, Jun. 2018.

[9] JM Wardlaw, C Smith, M Dichgans, "Small vessel disease: mechanisms and clinical implications," The Lancet Neurology, vol. 18, no. 7, pp. 684-696, Jun. 2019.

[10] MK Georgakis, M Duering JM Wardlaw, M Dichgansa, "WMH and long-term outcomes in ischemic stroke," Neurology, vol. 92, no. 12, pp. e1298-e1308, Mar. 2019.

[11] B Volbers, D Staykov, I Wagner et al., "Semi-automatic volumetric assessment of perihemorrhagic edema with computed tomography," Eur J Neurol, vol. 18, no. 11, pp. 1323-1328, Apr. 2011.

[12] A Charidimou, A Schmitt, D Wilson et al., "The Cerebral Haemorrhage Anatomical RaTing inStrument (CHARTS): Development and assessment of reliability" Journal of the Neurological 
Sciences, 2017 vol. 372 pp. 178-183

[13] JM Wardlaw, EE Smith, GJ Biessels et al., "Neuroimaging standards for research into small vessel disease and its contribution to ageing and neurodegeneration," The Lancet Neurology, vol. 12, no. 8, pp. 822-838, Jul. 2013.

[14] F Arba, G Mair, T Carpenter et al., "Cerebral White Matter Hypoperfusion Increases with SmallVessel Disease Burden. Data From the Third International Stroke Trial," Journal of Stroke and Cerebrovascular Diseases, vol. 26, no. 7, pp. 1506-1513, Jul. 2017.

[15] The IST-3 Collaborative group. The benefits and harms of intravenous thrombolysis with recombinant tissue plasminogen activator within $6 \mathrm{~h}$ of acute ischaemic stroke (the third international stroke trial [IST-3]): a randomized controlled trial. The Lancet 379, 2352-2363 (2012).

[16] MA Rodrigues, N Samarasekera, C Lerpiniere et al "The Edinburgh CT and genetic diagnostic criteria for lobar intracerebral haemorrhage associated with cerebral amyloid angiopathy: model development and diagnostic test accuracy study," The Lancet Neurology, vol. 17, no. 3, pp. 232-240, Feb. 2018.

[17] JC van Swieten, A Hijdra, PJ Koudstaal, J van Gijn, "Grading white matter lesions on CT and MRI: a simple scale.," J Neurol Neurosurg Psychiatry, vol. 53, no. 12, pp. 1080-1083, Dec. 1990.

[18] KGM Moons, JAH de Groot, W Bouwmeester et al., "Critical Appraisal and Data Extraction for Systematic Reviews of Prediction Modelling Studies: The CHARMS Checklist," PLoS Med, vol. 11, no. 10, pp. e1001744-13, Oct. 2014.

[19] R Ji, H Shen, Y Pan et al., "A novel risk score to predict 1-year functional outcome after intracerebral hemorrhage and comparison with existing scores," pp. 1-10, Dec. 2013.

[20] WD Tao, J Wang, G Schlaug, M Liu, MH Selim, “A Comparative Study of Fractional Anisotropy Measures and ICH Score in Predicting Functional Outcomes After Intracerebral Hemorrhage," Neurocritical Care, vol. 21, no. 3, pp. 417-425, Jun. 2014.

[21] M Stein, M Luecke, M Preuss, W Scharbrodt, A Joedicke, MF Oertel, “The Prediction of 30-Day Mortality and Functional Outcome in Spontaneous Intracerebral Hemorrhage with Secondary Ventricular Hemorrhage: A Score Comparison," in Trends in Neurovascular Surgery, vol. 112, no. 5, Vienna: Springer Vienna, 2011, pp. 9-11.

[22] JP Appleton, LJ Woodhouse, A Adami et al., "Imaging markers of small vessel disease and brain frailty, and outcomes in acute stroke," Neurology, vol. 94, no. 5, pp. e439-e452, Feb. 2020.

[23] YC Huo, Q Li, WY Zhang et al., "Total Small Vessel Disease Burden Predicts Functional Outcome in Patients With Acute Ischemic Stroke," Front. Neurol., vol. 10, pp. 483-8, Aug. 2019. 
[24] F Arba, GD Testa, N Limbucci et al., "Small vessel disease and clinical outcomes after endovascular treatment in acute ischemic stroke," pp. 1-9, Mar. 2019.

[25] MdC Valdes Hernandes, T Booth, C Murray et al. "Brain white matter damage in aging and cognitive ability in youth and older age". Neurobiology of Aging. 2013 Dec;34(12):2740-7.

[26] KB Lee, JS Kim, BY Hong, YD Kim, BY Hwang, SH Lim. "The Motor Recovery Related with Brain Lesion in Patients with Intracranial Hemorrhage”. Behavioural Neurology. 2015;2015(5-6):1-6.

[27] A Sreekrishan, AC Leasure, F-D Shi, DY Hwang et al., "Functional Improvement Among Intracerebral Hemorrhage (ICH) Survivors up to 12 Months Post-injury”. Neurocritical Care. 2017 Jul 6;27(3):326-33.

[28] J Puig, G Blasco, M Terceno et al., "Predicting Motor Outcome in Acute Intracerebral Hemorrhage," AJNR Am J Neuroradiol, vol. 40, no. 5, pp. 769-775, May 2019.

[29] LM Moura, R Luccas, JPQ de Paiva et al., "Diffusion Tensor Imaging Biomarkers to Predict Motor Outcomes in Stroke: A Narrative Review," Front. Neurol., vol. 10, pp. 641-17, May 2019.

[30] N Samarasekera, A Fonville, C Lerpiniere et al., "Influence of Intracerebral Hemorrhage Location on Incidence, Characteristics, and Outcome”. Stroke. 2015; 46:361-368 
Table 1. Baseline clinical characteristics, CT-based SVD biomarkers and outcomes

Variable

Age (mean)

Gender, female

Arterial Hypertension

Diabetes Mellitus

Atrial fibrillation

Hypercholesterolemia

Anticoagulant drug

Pre-ICH Cognitive Impairment $\$$

Glasgow Coma Scale score (mean)

ICH Location:

Deep

Lobar

Infratentorial

Intra-ventricular extension

ICH volume (mean)

White matter hypodensities

Yes*

No

Severe white matter hypodensities

Lacunes

Yes

No

Severe lacunes (lacunes $\geq 2$ )

Deep atrophy

None

Moderate

Severe

Cortical atrophy
Patients included in analysis $(\mathrm{N}=864)$

$$
\mathrm{N}(\%)
$$

73.6 (SD: 11.9)

$362(41.9)$

$580(67.1)$

$154(17.8)$

$301(34.8)$

$371(44.2)$

$345(39.9)$

$70(8.1)$

13.9 (SD: 1.9)

$429(49.6)$

361 (41.8)

74 (8.6)

256 (29.6)

14.9 (SD: 20.2)

443 (51.3)

421 (48.7)

$261(30.2)$

91 (10.5)

773 (89.5)

$18(2 \%)$

225 (26.0)

457 (52.9)

$182(21.1)$ 
None

Moderate

Severe

Severe atrophy\#

SVD burden score:

Non-severe (0-1)

Severe $(\geq 2)$

Neurosurgery performed

Pre-ICH mRS (median; IQR)

Pre- $\mathrm{ICH} \mathrm{mRS} \leq 2$

mRS at 6 months:
$132(15.3)$

$573(66.3)$

$159(18.4)$

258 (29.9)

$740(85.7)$

$124(14.3)$

$26(3.0)$

$0 ; 1$

$745(86.2)$

$156(18.0)$

$119(13.8)$

$54(6.3)$

$194(22.5)$

$76(8.8)$

$123(14.2)$

$142(16.4)$

6

SD, Standard Deviation; ICH, intracerebral haemorrhage; SVD, small vessel disease; mRS, modified Rankin Scale

$\mathcal{S}$ Pre-ICH cognitive impairment was considered present: (1) for those with a confirmed diagnosis of dementia prior to ICH, or (2) in case of Informant Questionnaire on Cognitive Decline in the Elderly $(I Q C O D E)>3.3$

${ }^{*} W M H$ present: if Van Swieten $\geq 1$ (either anterior or posterior)

\# Severe atrophy: if graded as severe in either deep or cortical regions 


\section{Figure 1. Patient flow chart}

\section{ICH, intracerebral hemorrhage.}

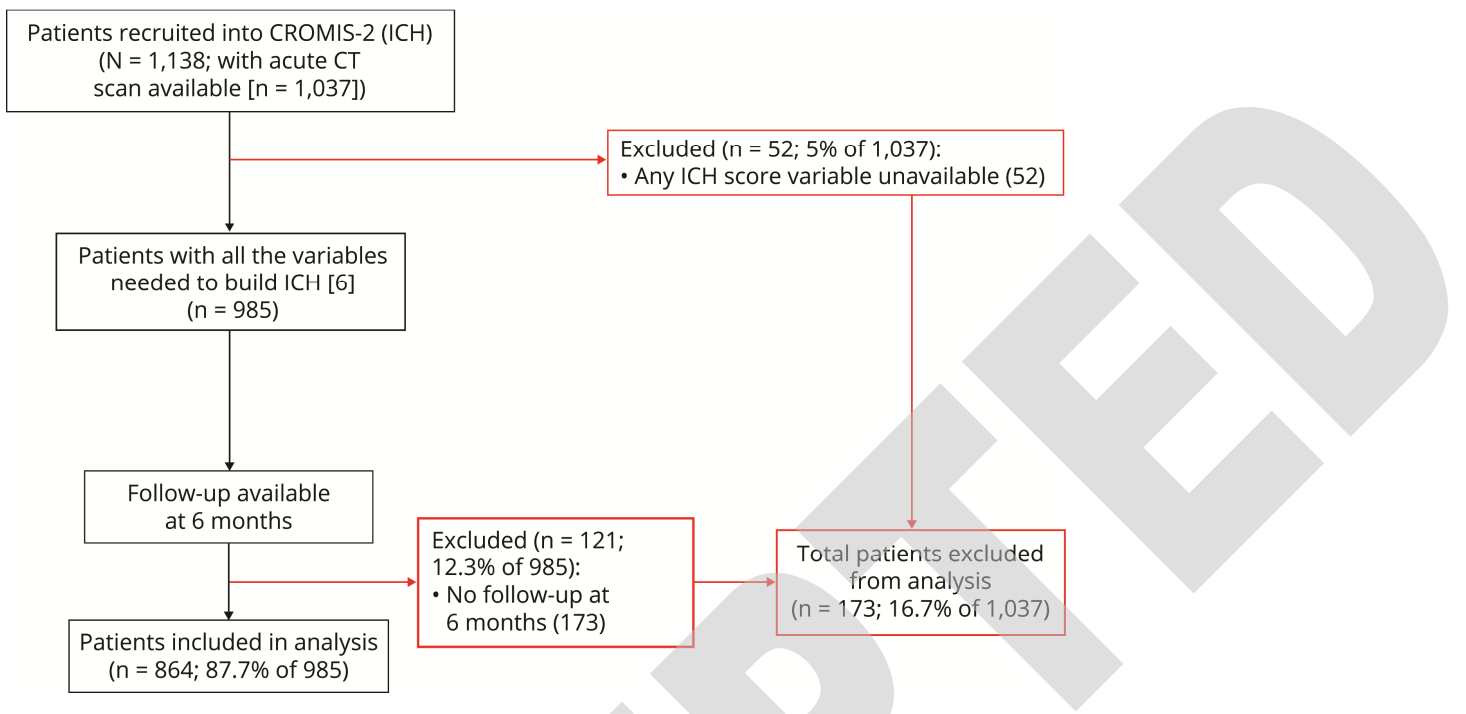

Figure 2. AUROC and Calibration Plot for ICH score

(A) AUROC for ICH score In Predicting Poor Functional Outcome (mRS 3 to 6) at 6 Months.

(B) Calibration Plot for ICH score According to Outcome Measurement Before and After

Recalibration. ICH, intracerebral haemorrhage; AUROC, area under the receiver operating curve; mRS, modified Rankin Scale.

A

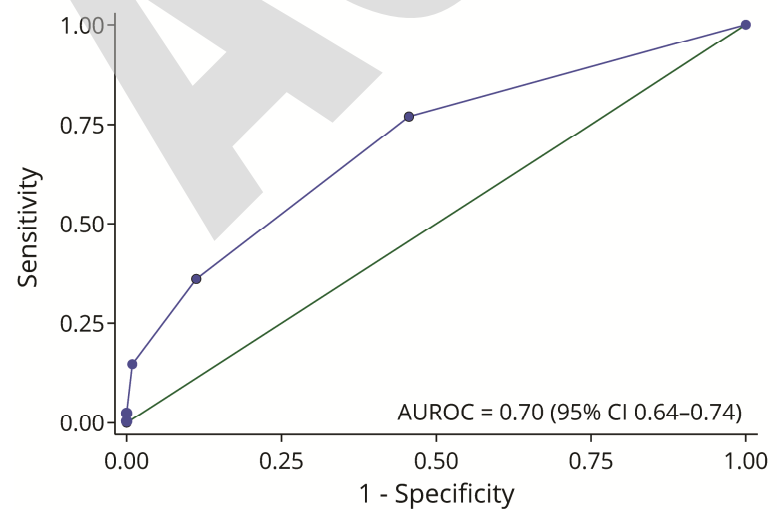

B $\quad$ o Original

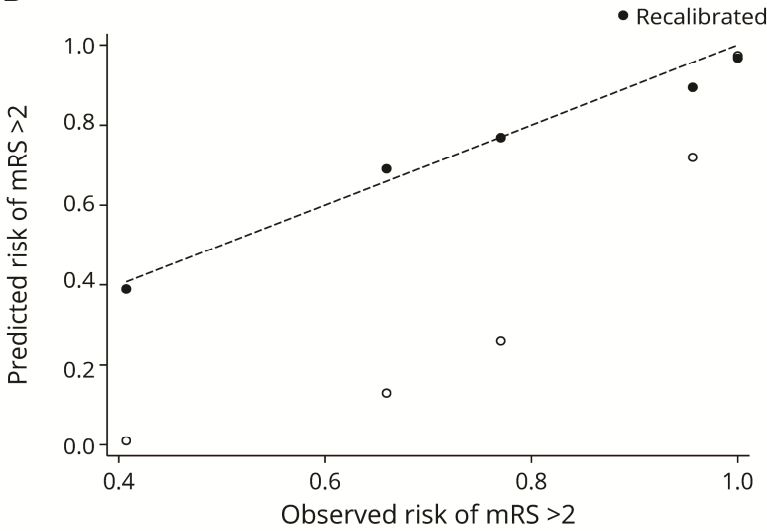


Table 2. Revised ICH score model. Multivariate analysis with calculation of $\beta$-coefficients and OR (and accordingly assigned integer score points) for original variables included in ICH score.

\begin{tabular}{|c|c|c|c|}
\hline Variables & Points & $\beta$-coefficients & OR $(95 \% \mathrm{CI})$ \\
\hline GCS & & 1.24 & $3.44(1.99-5.93)$ \\
\hline *3 to 12 & 2 & & \\
\hline 13 to 15 & 0 & & \\
\hline ICH Volume & & 1.26 & $3.54(2.02-6.20)$ \\
\hline$\geq 30 \mathrm{cc}$ & 2 & & \\
\hline$<30 \mathrm{cc}$ & 0 & & \\
\hline ICH Location & & & $1.71(1.00-2.91)$ \\
\hline Infra-tentorial & 1 & & \\
\hline Supra-tentorial & 0 & & \\
\hline Age & & & $3.02(2.16-4.21)$ \\
\hline$\geq 80$ & 2 & & \\
\hline$<80$ & 0 & & \\
\hline IVH & & 0.57 & $1.77(1.25-2.50)$ \\
\hline Yes & 1 & & \\
\hline No & 0 & & \\
\hline \multicolumn{4}{|c|}{ Score Interpretation } \\
\hline & Points & \multicolumn{2}{|c|}{ Poor $(\mathrm{mRS}>2)$ 6-months outcome rate } \\
\hline & 0 & \multicolumn{2}{|c|}{$42 \%$} \\
\hline & 1 & \multicolumn{2}{|c|}{$56 \%$} \\
\hline & 2 & \multicolumn{2}{|c|}{$69 \%$} \\
\hline & 3 & \multicolumn{2}{|c|}{$80 \%$} \\
\hline & 4 & \multicolumn{2}{|c|}{$89 \%$} \\
\hline & 5 & \multicolumn{2}{|c|}{$94 \%$} \\
\hline & 6 & \multicolumn{2}{|c|}{$96 \%$} \\
\hline & $7-8 \S$ & \multicolumn{2}{|c|}{$98 \%$} \\
\hline
\end{tabular}


*only 5 patients in our cohort had a of GCS 3 or 4 (excluded);

$\mathcal{S}$ Only one patient with score 8;

GCS, Glasgow Coma Scale; ICH, intracerebral haemorrhage; IVH, intra-ventricular extension; WMH, white matter hypodensities; $S V D$, small vessel disease

Table 3. Clinical and radiological characteristics in the entire cohort and univariable associations with poor 6-month functional outcome (mRS >2)

$\begin{array}{cc}\mathrm{N}(\%) & \text { Poor outcome } \\ (\mathrm{mRS}>2) & \text { OR }(95 \% \mathrm{CI})\end{array}$

Clinical Variables

Age:

$3.06(2.22-4.22)<0.001$

$>80$

$<80$

$295(34.1) \quad 230(78.0)$

$<80$

$569(65.9)$

305 (53.6)

GCS:

3 to $4^{*}$

5 to 12

$4(0.4)$

$4(100.0)$

13 to 15

$130(15.1)$

$112(86.2)$

$4.7(2.81-7.85)$

$<0.001$

730 (84.5)

$419(57.4)$

ICH volume:

4.7 (2.76-7.99)

$<0.001$

$<30$

$738(85.4)$

$426(57.7)$

$\geq 30$

$126(14.6) \quad 109(86.5)$

Intraventricular extension:

$2.19(1.59-3.03) \quad<0.001$

Yes

No

256 (29.6) $190(74.2)$

608 (70.4) $345(56.7)$

Location:

$1.31(0.79-2.18) \quad 0.296$

Supratentorial

$790(91.4) \quad 485(61.4)$

Infratentorial

$74(8.6) \quad 50(67.6)$

CT SVD biomarkers

White Matter Hypodensities

$1.80(1.36-2.37) \quad<0.001$

$\begin{array}{lll}\text { Present } & 443(51.3) & 304(68.6) \\ \text { Absent } & 421(48.7) & 231(54.9)\end{array}$

Lacunes:

$1.09(0.69-1.71)$

0.706

Present

$91(10.5)$

$58(63.7)$ 
Absent

Severe WMH

Yes

No

Severe lacunes (lacunes $\geq 2$ )

Yes

No

Cortical Atrophy:

None

Moderate

Severe

Deep Atrophy:

None

Moderate

Severe

Severe Atrophy:

Yes

No

Severe SVD burden score:

Non-Severe (0-1)

Severe (2-3) §
$773(89.5)$

$477(61.7)$

$1.48(1.09-2.01)$

$261(30.2) \quad 178(68.2)$

0.012

$603(69.8) \quad 357(59.2)$

$1.24(0.46-3.32)$

$18(2.1) \quad 12(66.7)$

$846(97.9) \quad 523(61.8)$

$132(15.3) \quad 60(45.5)$

$573(66.3) \quad 350(61.1)$

$1.88(1.29-2.76)$

0.001

$159(18.4) \quad 125(78.6)$

$4.41(2.65-7.35) \quad<0.001$

$225(26.0) \quad 111(49.3)$

$457(52.9) \quad 285(62.4)$

$1.70(1.23-2.35)$

0.001

$182(21.1)$

139 (76.4)

$3.32(2.16-5.11)$

$<0.001$

$2.36(1.70-3.26)$

$258(29.9)$

$194(75.2)$

$<0.001$

$606(70.1) \quad 341(56.3)$

$1.84(1.20-2.81)$

$740(85.6) \quad 444(60.0)$

$124(14.4) \quad 91(73.4)$

0.004

* only 5 patients in our cohort had a GCS of 3 or 4 (excluded for OR evaluation)

$\mathcal{S}$ only 4 patients in our cohort with SVD score $=3$ (excluded for OR evaluation)

AUROC, area under the curve; GCS, Glasgow Coma Scale; ICH, intracerebral haemorrhage; IVH, intra-ventricular extension; $W M H$, white matter hypodensities; $S V D$, small vessel disease; $O R$, odds ratio; 95\% CI, 95\% confidence interval. 
Table 4. Association of SVD biomarkers and severe SVD burden score with poor functional outcome (mRS >2) at 6 months. Models are adjusted for ICH score variables: age, GCS, ICH volume, Intraventricular extension, ICH location.

\begin{tabular}{rcc}
\hline WMH presence & OR $(95 \% \mathrm{CI})$ & P value \\
Lacunes presence & $1.52(1.12-2.06)$ & 0.007 \\
Cortical atrophy presence & $1.80(1.19-2.73)$ & 0.268 \\
Deep atrophy presence & $1.66(1.17-2.34)$ & 0.006 \\
Severe WMH & $1.29(0.93-1.81)$ & 0.004 \\
Severe atrophy & $1.94(1.36-2.74)$ & 0.132 \\
Severe SVD burden score & $1.57(0.99-2.48)$ & $<0.001$ \\
\hline
\end{tabular}

GCS, Glasgow Coma Scale; ICH, intracerebral haemorrhage; IVH, intra-ventricular extension; WMH, white matter hypodensities; SVD, small vessel disease; OR, odds ratio; 95\% CI, 95\% confidence interval. 
Table 5. Sequential addition to ICH score of dichotomized CT-based markers of SVD significantly related to outcome.

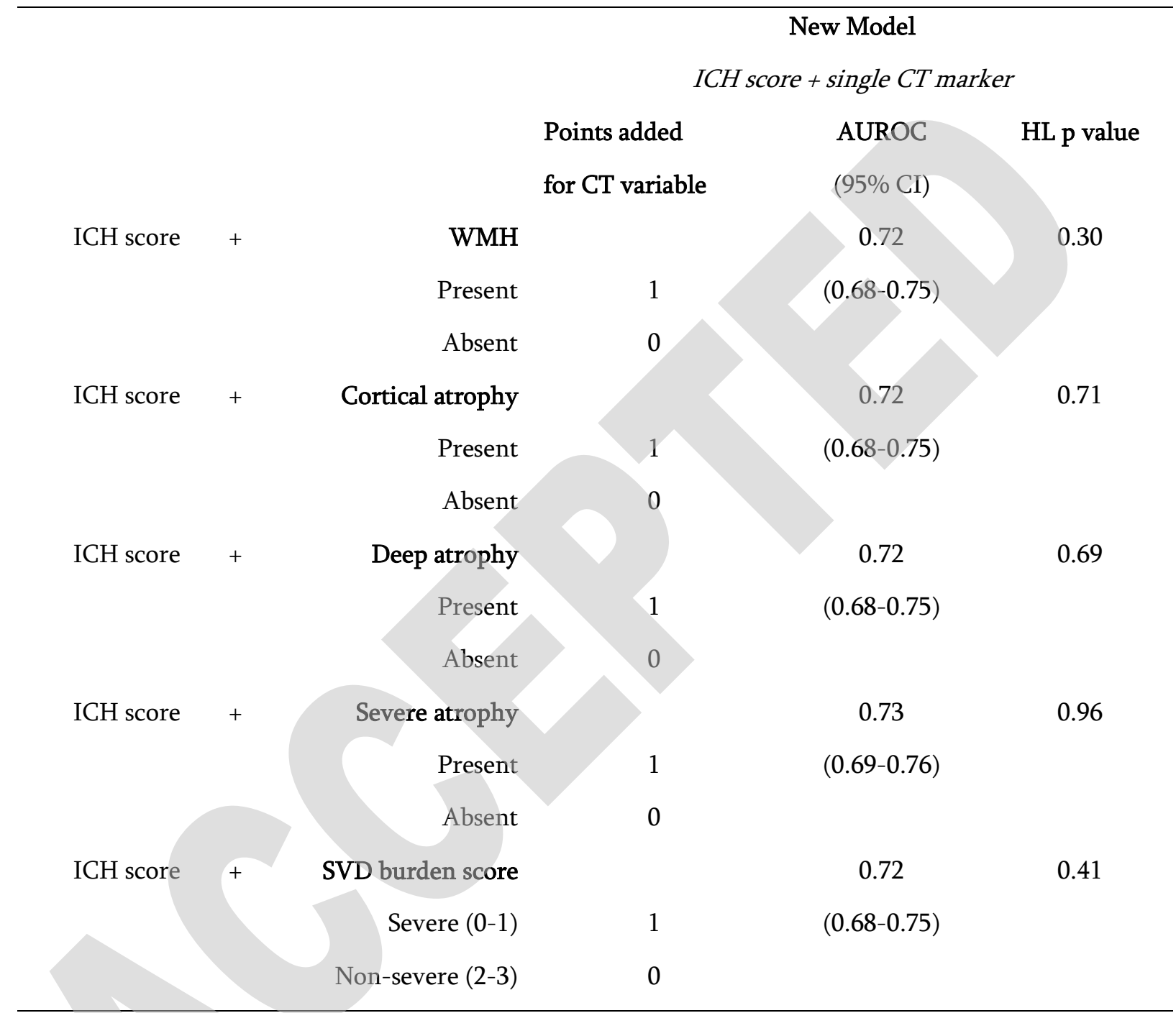

AUROC area under the receiver operating characteristic; GCS, Glasgow Coma Scale; ICH, intracerebral haemorrhage; IVH, intra-ventricular extension; $W M H$, white matter hypodensities; $S V D$, small vessel disease; HL, Hosmer-Lemeshow test. 


\title{
Neurology
}

\author{
Cerebral Small Vessel Disease and Functional Outcome Prediction after Intracerebral \\ Haemorrhage \\ IC Hostettler, G Schwarz, G Ambler, et al. \\ Neurology published online February 24, 2021 \\ DOI 10.1212/WNL.0000000000011746
}

This information is current as of February 24, 2021

Updated Information \&

Services

Subspecialty Collections

Permissions \& Licensing

Reprints including high resolution figures, can be found at:

http://n.neurology.org/content/early/2021/02/24/WNL.0000000000011746.f ull

This article, along with others on similar topics, appears in the following collection(s):

All Cerebrovascular disease/Stroke

http://n.neurology.org/cgi/collection/all_cerebrovascular_disease_stroke CT

http://n.neurology.org/cgi/collection/ct

Intracerebral hemorrhage

http://n.neurology.org/cgi/collection/intracerebral_hemorrhage

Prognosis

http://n.neurology.org/cgi/collection/prognosis

Information about reproducing this article in parts (figures,tables) or in its entirety can be found online at:

http://www.neurology.org/about/about_the_journal\#permissions

Information about ordering reprints can be found online:

http://n.neurology.org/subscribers/advertise

Neurology ${ }^{\circledR}$ is the official journal of the American Academy of Neurology. Published continuously since 1951, it is now a weekly with 48 issues per year. Copyright (C) 2021 American Academy of Neurology. All rights reserved. Print ISSN: 0028-3878. Online ISSN: 1526-632X.

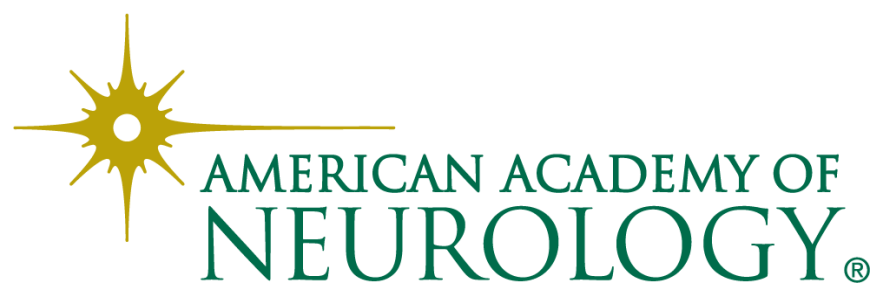

